\title{
Tolerance of potato cultivars to potato spindle tuber viroid PSTVd
}

Khiutti A.V. ${ }^{1 *}$, Mironenko N.V. ${ }^{1}$, Lashina N.M. ${ }^{1}$, Matsushita Y. ${ }^{2}$, Afanasenko O.S. ${ }^{1}$

${ }^{1}$ All-Russian Institute of Plant Protection, St. Petersburg, Russia

${ }^{2}$ NARO, Institute of Vegetable and Floriculture Science Tsukuba, Japan

*email:alexanderkhyutti@mail.ru

Potato spindle tuber viroid (PSTVd) has a wide geographical distribution. The disease has quarantine status and is highly harmful. PSTVd infected potato plants become stunted, their leaves turn yellow, and infected tubers become small and cracked. The genotype of host and PSTVd strain affects the manifestation of symptoms. The aim of the research was to determine the tolerance of modern potato cultivars to two strains of PSTVd. Tolerance of 10 potato cultivars of Russian breeding - Aurora, Bison, Udacha, Elizabeth, Kinza, Mirage, Ariel, Sapsan, Favorite and Armada and 12 foreign cultivars - Red Scarlett, Riviera, Gala, Colomba, Lugovskoy, Odyssey, Adretta, Red Fantasy, Queen Anne, Berkut, Nazca and Mandola to strains PSTVd VP87 and VP35 has been determined. One step RT-PCR with primer set P3/P4 was used for detection PSTVd in the potato leaves. 2 months after the inoculation, the diagnostic fragments indicating the presence of the PSTVd were found in plants of all cultivars except Elizabeth, Favorite and Sapsan (data from two biological repetitions). In cultivar Elizabeth after 2.5 months of inoculation PSTVd was also not detected, but after 3.5 months, the diagnostic fragment indicating a replication of the viroid was determined. Slow, in comparison with other cultivars of viroid replication dynamics may be one of the signs of tolerance of this cultivar. A group of strongly affected varieties was distinguished, in which very bright diagnostic fragments were determined to both strains in both repetitions of the experiment, indicating a high concentration of viroid. These are cultivars Udacha, Gala, Red Scarlet, Bison, and Colombo. The symptoms of the disease on different cultivars was studied. The main symptoms were wrinkling, twisting of leaves and deformation of the leaf tip. Brightly expressed symptoms of viroid on formed tubers manifested in elongated form and deformations were noted on cultivars Udacha and Aurora.

Acknowledgements: The research was supported by a grant from the Russian Science Foundation No. 20-46-07001. The Strategic International Collaborative Research Project promoted by the Ministry of Agriculture, Forestry and Fisheries, Tokyo, Japan (No. J008837). 